Cahiers
de a Recherche
sur les Droits Fondamentaux

\section{Cahiers de la recherche sur les droits} fondamentaux

$7 \mid 2009$

L'universalisme des droits en question(s). La Déclaration universelle des Droits de l'homme, 60 ans après

\title{
La gestion de la pluralité des systèmes juridiques par les États d'Afrique noire : les enseignements de l'expérience camerounaise
}

\section{Pierre-Étienne Kenfack}

\section{OpenEdition \\ Journals}

\section{Édition électronique}

URL : https://journals.openedition.org/crdf/6717

DOI : $10.4000 /$ crdf. 6717

ISSN : 2264-1246

\section{Éditeur}

Presses universitaires de Caen

\section{Édition imprimée}

Date de publication : 31 décembre 2009

Pagination : 153-160

ISBN : 978-2-84133-342-4

ISSN : $1634-8842$

\section{Référence électronique}

Pierre-Étienne Kenfack, «La gestion de la pluralité des systèmes juridiques par les États d'Afrique noire : les enseignements de l'expérience camerounaise », Cahiers de la recherche sur les droits fondamentaux [En ligne], 7 | 2009, mis en ligne le 15 décembre 2020, consulté le 14 novembre 2022 URL : http://journals.openedition.org/crdf/6717 ; DOI : https://doi.org/10.4000/crdf.6717 


\title{
La gestion de la pluralité des systèmes juridiques par les États d'Afrique noire: les enseignements de l'expérience camerounaise ${ }^{1}$
}

\author{
Pierre-Étienne KENFACK \\ Enseignant à I'Université de Yaoundé 2-Soa (Cameroun)
}

I. La piste à éviter : la suppression de la pluralité

A. L'impossible mise à l'écart d'un système juridique

B. L'impossibilité d'imposer un système juridique

II. La voie à explorer : I'exploitation de la pluralité

A. Le choix d'une technique d'exploitation de la pluralité

B. Mise en œuvre de la technique choisie

Y a-t-il encore de la place pour une étude sur le pluralisme juridique et, en particulier, pour une des ses importantes manifestations, la pluralité des systèmes juridiques dans un État, spécialement d'Afrique noire? Plusieurs arguments peuvent militer en faveur d'une réponse négative à cette interrogation. Des auteurs éminents ont tellement écrit sur la question du pluralisme juridique qu'il peut paraître présomptueux de revenir sur le sujet ${ }^{2}$. Relativement à la gestion de la pluralité des systèmes par les États d'Afrique noire, la question préoccupe les auteurs depuis les indépendances ${ }^{3}$ de sorte qu'on peut considérer le domaine comme un champ désormais aride. Mais des

1. Je remercie le professeur Thierry Le Bars de l'Université de Caen Basse-Normandie dont les conseils et l'encadrement ont permis la réalisation du présent article.

2. De manière non exhaustive: J. Gilissen (dir.), Le pluralisme juridique, Bruxelles, Éditions de l’Université de Bruxelles, 1972 ; J. Griffith, « What is legal pluralism? ", Journal of Legal Pluralism and Unofficial Law, ${ }^{\circ}$ 24, 1986, p. 1 sq. ; J.-L. Bergel, Le plurijuridisme, Aix-en-Provence, PUAM, 2005; A. Soeteman, Pluralism and Law, Amsterdam, Springer, 2001; Laboratoire d'anthropologie juridique de Paris, Les pluralismes juridiques. Cahiers d'Anthropologie du droit, Paris, Éditions Karthala, 2003; Le pluralisme, Archives de Philosophie du droit, n 49, 2005; J. Kellerhals, D. Manaï et R. Roth, Pour un droit pluriel. Études offertes au professeur Jean-François Perrin, Genève - Bâle - Munich, Helbing \& Lichtenhahn (Collection genevoise), 2002; P. Lampue, «L'influence du droit français et du droit coutumier sur les lois civiles africaines », in Dynamiques et finalités des droits africains, G. Conac (dir.), Paris, Economica, 1980, p. 14 sq.

3. Voir notamment: G. A. Kouassigan, Quelle est ma loi ? Tradition et modernisme dans le droit privé de la famille, Paris, Pédone, 1974; A. Sow-Sidibe, Le pluralisme juridique en Afrique (l'exemple du droit successoral sénégalais), Paris, LGDJ (Bibliothèque africaine et malgache, t. 50), 1991; M. NkouendjinYotnda, «Soliloques sur certains problèmes soulevés par l'organisation judiciaire de la République du Cameroun », Penant, $\mathrm{n}^{\circ}$ 751, 1976, Doctrine, p. 5 sq.; P. Lampue, «Droit écrit et droit coutumier en Afrique francophone», Penant, $\mathrm{n}^{\circ} 763,1979$, p. 245 sq.; P. L. Agondjo-Okawe, «Domaines d'application des droits traditionnels ", in Encyclopédie juridique de l'Afrique, t. 1, L'État et le droit, P. Tousard (dir.), Abidjan, Nouvelles Éditions africaines, 1982 , p. 393 sq.; P. Bourel, «Les conflits de droits », in Encyclopédie juridique de l'Afrique, t. 1, L'État et le droit, p. 423 sq.; R. Degni-Segui, « Codification et uniformisation du droit », in Encyclopédie juridique de l'Afrique, t. 1, L'État et le droit, p. 45 sq.; S. Melone, «Du bon usage du pluralisme judiciaire en Afrique ", Revue camerounaise de droit (RDC), $\mathrm{n}^{\circ} 31,1986$, p. 5 sq. 
arguments en faveur d'une réponse affirmative peuvent également être mis en évidence. En effet, bien qu'ancien, le pluralisme juridique est une problématique constamment renouvelée: il admet et sans doute admettra toujours de nouvelles recherches ${ }^{4}$. Quant à la gestion de la pluralité des systèmes juridiques en Afrique, il est possible de revenir sur le sujet pour plusieurs raisons dont deux majeures au moins. D'après la première, la plupart de ces études traitent soit des aspects particuliers de la pluralité des systèmes juridiques ${ }^{5}$, soit de l'application de la pluralité à un domaine spécifique ${ }^{6}$. Quant aux rares études embrassant l'ensemble de la question, datant des lendemains des indépendances, elles ne pouvaient se préoccuper des aspects qui ne se sont révélés que plus tard et encore moins tirer des conséquences des mécanismes à peine mis en place. D'après la seconde, la quasi-totalité des études ont surtout abordé la question sous l'angle de la présentation de la gestion, en répondant prioritairement à la question de savoir comment est géré le pluralisme.

Si on change de perspective d'analyse pour envisager la question sous l'angle de la pertinence des solutions en usage, sous l'angle des enseignements à tirer du système actuel de gestion, la question se renouvelle et peut mériter de nouvelles études. C'est dans cette perspective que nous situons cette recherche centrée sur le Cameroun. Le choix de ce pays s'explique par sa soumission pendant la période coloniale au mandat, puis à la tutelle de la France et de la Grande-Bretagne, avec pour conséquence sur le plan juridique, la cohabitation de trois systèmes juridiques. L'étude sera également centrée sur le droit privé parce que seule cette branche du droit tolère une pluralité de systèmes sur un même territoire.

Pour bien situer le débat, il est important de clarifier la notion de système juridique, expression qui ne fait pas l'objet d'une définition partagée par les auteurs. Le Vocabulaire juridique de l'Association Henri Capitant donne au mot «système» trois sens dont deux permettent de clarifier celui de l'expression «système juridique ». D'après l'un, le système désigne un ensemble coordonné, ou l'agencement d'un ensemble. D'après l'autre, il désigne un ensemble de règles, considéré sous le rapport de ce qui en fait la cohérence ${ }^{7}$. La combinaison de ces deux sens permet de dire que le système juridique désigne le droit d'un État, d'une communauté autonome ou d'une société territoriale ou personnelle, constitué des règles et des modalités de leur mise en œuvre, formant un ensemble cohérent ${ }^{8}$.

Cette définition permet de réaliser que les États d'Afrique noire, dont la création est le résultat d'une mise ensemble, d'autorité, par les puissances coloniales des portions de territoires et des populations ayant des systèmes juridiques différents, sont nés avec plusieurs systèmes juridiques : celui ou ceux introduits par la ou les puissances coloniales et celui en vigueur avant leur arrivée et qu'on a qualifié de «droits traditionnels africains " 9 .

La première préoccupation a été de savoir ce qu'il fallait faire de ces systèmes susceptibles de s'appliquer au même moment à des problèmes similaires. La réponse eut été simple si chaque système s'était préoccupé des problèmes distincts de ceux des autres. Mais c'était des systèmes concurrents et on se trouvait dès lors en présence d'une des déclinaisons du problème général de pluralisme juridique $^{10}$.

Après avoir géré cette pluralité avec plus ou moins de bonheur, les puissances coloniales, mandatrices ou tutrices, l'ont transmise aux États africains lors des indépendances. Tandis que la plupart des pays héritaient d'un pluralisme composé de deux grands systèmes, le Cameroun placé successivement sous mandat, puis sous la tutelle de la France et de l'Angleterre, hérita d'un pluralisme composé de trois systèmes: le système germanoromain introduit par la France, le système de la Common Law introduit par la Grande-Bretagne et celui des droits traditionnels africains. Alors que les deux premiers répondent par leur structure et leurs règles de fonctionnement aux exigences universelles de l'État nation, les systèmes de droits traditionnels sont ceux dont les modes de formation et de légitimation ne se préoccupent nullement de la notion d'État ${ }^{11}$. Échappant au monisme étatique, ils se caractérisent par leur rattachement à un groupe, une ethnie ou à un espace géographique sur le territoire ${ }^{12}$. Comme on l'a écrit avec pertinence, ce sont « des systèmes

4. Parmi les travaux récents sur la question, lire notamment: L. Fontaine (dir.), Droit et pluralisme, Bruxelles, Bruylant (Droit et justice, $\mathrm{n}^{\circ} 76$ ), 2008; E. Leroy, «Le pluralisme juridique aujourd'hui ou l'enjeu de la juridicité», Les pluralismes. Cahiers d'Anthropologie du droit, p. 7 sq. ; J. Vanderlinden, "Vers une nouvelle conception du pluralisme juridique», RRJ Droit prospectif, n 2, 1993, p. 573 sq.; J. Vanderlinden, "Trente ans de longue marche sur la voie du pluralisme juridique», in Les pluralismes juridiques, p. 21 sq.; H. Lamine, "Pour un pluralisme juridique plus effectif», in Les pluralismes juridiques, p. 157 sq. ; V. Petev, «Pluralisme juridique, construction européenne et droits participatifs », in Le pluralisme, p. 47 sq.; D. Terre, «Le pluralisme et le droit», in Le pluralisme, p. 69 sq.

5. P. Lampue, «Droit écrit et droit coutumier en Afrique francophone»; P. L. Agondjo-Okawe, «Domaines d'application des droits traditionnels»; S. Melone, «Du bon usage du pluralisme judiciaire en Afrique».

6. A. Sow Sidibe, Le pluralisme juridique en Afrique (l'exemple du droit successoral sénégalais), Paris, LGDJ (Bibliothèque africaine et malgache), t. 50 , 1991.

7. G. Cornu, Vocabulaire juridique de l'Association Henri Capitant, Paris, PUF (Quadrige), 2007, p. 906 sq.

8. D. Alland et S. Rials (dir.), Dictionnaire de la culture juridique, Paris, Lamy - PUF (Quadrige), 2003, p. 1462 sq. Pour ces auteurs, le système est un ensemble complexe, constitué des parties liées entre elles par des relations stables.

9. E. Leroy et M. Wane, «La formation des droits non étatiques», in Encyclopédie juridique de l’Afrique, t. 1, L'État et le droit, p. 353 sq.

10. Par-delà les controverses, nous entendons par pluralisme juridique la coexistence sur un territoire donné, à un même moment, de plusieurs ordres juridiques distincts plus ou moins dépendants les uns des autres et éventuellement concurrents. Cf. D. Alland et S. Rials (dir.), Dictionnaire de la culture juridique, p. 1159. Sur l'évolution du sens du concept de pluralisme juridique, voir D. Terre, «Le pluralisme et le droit », p. 69 sq.; J. Vanderlinden, "Vers une nouvelle conception du pluralisme juridique », p. 573 sq.

11. Ibid., p. 575

12. Cf. M. Kamto, «Une justice entre tradition et modernité», J. du Bois de Gaudusson et G. Conac (dir.), La justice en Afrique. Afrique contemporaine, $\mathrm{n}^{\circ} 156$ (spécial), 1990, p. 57 sq. 
juridiques issus de l'expérience précoloniale, mais appliqués depuis lors dans des instances de règlement des conflits dont ni les modes de formation ni les modes de légitimation ne sont déterminés par l'État colonial ou post-colonial $»^{13}$.

Depuis l'accession à l'indépendance, au lieu de remettre en cause cette pluralité de systèmes juridiques, le Cameroun a développé des techniques et mécanismes permettant de la gérer. Quelles leçons, quels enseignements peut-on tirer de son expérience ? C'est le principal objectif de cette recherche qui, au-delà d'un bilan, vise la recherche des pistes pour une meilleure gestion de la pluralité des systèmes juridiques par les États d'Afrique noire.

De l'expérience du Cameroun se dégagent deux pistes contradictoires pouvant aider un État à gérer avec des chances de succès la pluralité de ses systèmes juridiques. Une à éviter : la suppression de la pluralité (I) ; une autre à explorer : son exploitation (II).

\section{La piste à éviter : la suppression de la pluralité}

Pour gérer la pluralité des systèmes juridiques, n'estil pas plus simple de supprimer la source de difficulté, de faire disparaittre la pluralité? On peut atteindre un tel résultat en mettant à l'écart le ou les systèmes perturbateurs, indésirables. On peut également y parvenir en imposant un système sans se préoccuper de l'existence des autres qui automatiquement disparaîtront. Le Cameroun a expérimenté les deux possibilités sans pouvoir parvenir au résultat souhaité. L’expérience de ce pays permet de réaliser que la suppression de la pluralité est une piste à éviter dans la recherche des solutions de gestion de la pluralité des systèmes juridiques car, ni la mise à l'écart (A), ni l'imposition (B) d'un système juridique ne sont possibles.

\section{A. L'impossible mise à l'écart d'un système juridique}

Peut-on gérer la pluralité des systèmes par la mise à l'écart de certains? Les puissances coloniales l'ont pensé et ont entrepris des démarches dans ce sens, la cible étant le système des droits traditionnels, mais toutes ces tentatives se sont soldées par des échecs ${ }^{14}$.

À l'accession à l'indépendance, le débat a ressurgi au sein des pays aspirant à la modernité. Partant de ce que les coutumes africaines sont un obstacle à la marche vers la modernité, certains pays ont proclamé leur suppression ${ }^{15}$. Ayant reçu un héritage composé de systèmes assez différents et, sans doute, échaudées par l'échec des puissances coloniales, les autorités camerounaises ont choisi la voie douce. Tout en proclamant et en organisant la cohabitation des systèmes sur le territoire, elles ont entrepris de faire disparaître progressivement le système de droit coutumier.

Le ton est donné par le législateur et les autorités administratives à travers la création d'une école de formation des magistrats qui ne fait pas figurer les enseignements de droit coutumier dans son programme, à travers l'affectation des magistrats formés dans ces écoles à la tête des juridictions de droit traditionnel et surtout à travers l'arbitrage du conflit de compétence entre les juridictions de droit traditionnel et les juridictions de droit moderne. Tandis que lorsqu'elles sont saisies, les juridictions de droit moderne qui appliquent le droit d'inspiration étrangère doivent automatiquement trancher le litige, celles de droit traditionnel qui appliquent la coutume ne peuvent le faire que si les deux parties sont d'accord. C'est ce qui ressort de l'article 2 du Décret $n^{\circ}$ 69/DF/544 du 19 décembre 1969 fixant l'organisation judiciaire et la procédure devant les juridictions traditionnelles du Cameroun oriental qui dispose que la compétence des juridictions traditionnelles est subordonnée à l'acceptation de toutes les parties en cause. La Cour suprême du Cameroun l'a expressément rappelé dans un arrêt rendu le 7 avril 1983 en ces termes : « [...] la compétence des juridictions traditionnelles est subordonnée à l'acceptation des parties [...] en conséquence, dès que le déclinatoire de compétence est régulièrement soulevé par l'une des parties en cause devant le juge coutumier, celui-ci est obligé de se dessaisir au profit de la juridiction de droit moderne ${ }^{16}$.» Un déclinatoire de compétence qui doit se faire in limine litis entraînant automatiquement compétence de la juridiction de droit moderne ${ }^{17}$.

Prenant le relais, la jurisprudence a posé un certain nombre de règles qui, jusqu'à ce jour, permettent d'écarter la coutume devant les juridictions de droit traditionnel.

13. M. Alliot, «L'État et la société en Afrique noire: greffes et rejets ", in Actes du colloque État et société en Afrique noire, Paris, septembre 1980, Paris, Société française d'histoire d'outre-mer, 1983, p. 9; F. Synder, "Droit non étatique et législation nationale au Sénégal», in Dynamiques et finalités des droits africains, Paris, Economica, 1980, p. 259 sq.; E. Leroy et M. Wane, «La formation des droits "non étatiques" ", in Encyclopédie juridique de l’Afrique, t. 1, L'État et le droit, p. 353 sq.

14. Lire notamment: M. Tataw, «Où en est-on avec le droit de la famille au Cameroun?», Penant, n ${ }^{\circ}$ 52, 2005, p. 347 sq.; S. Ombiono, « Le mariage coutumier dans le droit positif camerounais", Penant, $n^{\circ}$ 799, 1989, p. 32 sq.; R. Degni-Segui, "Codification et unification du droit ", in Encyclopédie juridique de l'Afrique, t. 1, L'État et le droit, p. 453 sq.; J. Hilaire, "Variations sur le mariage, à propos de la codification en Afrique noire ", Penant, ${ }^{\circ}$ 719, 1968 , p. 147 sq.

15. La Guinée directement, par l'ordonnance n ${ }_{45}$ PRG du 20 novembre 1960 dont l'article 5 proclame l'abolition de la coutume, la Côte-d'Ivoire, le Mali, le Burundi et le Rwanda indirectement par la suppression des tribunaux coutumiers. Lire sur ce point, S. Melone, «Du bon usage du pluralisme judiciaire en Afrique ", p. 5 sq.

16. C.S. arrêt $\mathrm{n}^{\circ} 49 / \mathrm{L}$ du 7 avril 1983, Revue camerounaise de droit, $\mathrm{n}^{\circ} 25,1983, \mathrm{p} .69$.

17. Cf. article 2 du Décret $n^{\circ}$ 69/DF/544 du 19 décembre 1969 fixant l'organisation judiciaire et la procédure devant les juridictions traditionnelles du Cameroun oriental qui pose que «nonobstant toutes dispositions contraires, la juridiction de droit moderne devient compétente dans le cas où l'une des parties décline la compétence d'une juridiction de droit traditionnel ». 
Dès 1963 et par la suite à plusieurs reprises, la Cour suprême du Cameroun a proclamé que dans les matières où il a été légiféré, la loi l'emporte sur la coutume ${ }^{18}$. Or, les lois, aussi bien pré que post-indépendance, ont été élaborées à partir d'une volonté de mettre à l'écart la coutume jugée contraire à l'idéologie de développement ${ }^{19}$. C'était une brèche importante dans l'édifice de la coutume qui laissait entrevoir sa disparition prochaine, car, le pays étant au début de son processus de production de lois, il était évident que plus les lois allaient se multiplier, plus la coutume allait perdre de son influence. Elle a, par la suite, proclamé qu'en cas de silence, d'obscurité de la coutume, ou de contrariété de celle-ci à l'ordre public, la loi devait être appliquée. L'entreprise de négation de la coutume amorcée par le législateur recevait par cette jurisprudence un renfort important car, la notion d'ordre public, étant non définie et fluctuante, devait permettre au juge d'écarter très souvent la coutume au profit du droit écrit.

Cette technique de gestion du pluralisme a-t-elle produit les résultats escomptés? Les systèmes de droit traditionnel ont-ils disparu ou sont-ils en voie de disparition?

Il y a lieu d'en douter, car en marge du système juridique étatique, malgré l'existence des juridictions traditionnelles, continuent de fonctionner des systèmes de droits traditionnels, organisés autour des communautés ethniques. Bien que présentant des variantes parce que tenant compte des conceptions du monde, des échelles de valeurs et des modes d'organisation sociale et politique de chaque ethnie, ces systèmes, qui forment de véritables «sous-ordres juridiques dans l'espace étatique ${ }^{20}$, ont des caractéristiques communes.

Ils relativisent l'importance des formes et de la procédure et utilisent une langue connue de ceux qui y recourent. Ils combinent des règles juridiques avec la morale et la religion ${ }^{21}$. Les juges qui y sont souvent des autorités traditionnelles, des patriarches ou des anciens y apparaissent plus comme des conciliateurs soucieux de distribuer équitablement le blâme et la louange ${ }^{22}$. Ils ont pour mission de protéger l'ordre social, de préserver l'ordre social plutôt que de déterminer les droits individuels des justiciables en fonction de la règle de droit applicable au cas d'espèce. La fin du procès dans ces systèmes est généralement un geste symbolique de réconciliation ${ }^{23}$.

Ils ont la préférence de la plupart des citoyens des zones rurales et de certains citoyens des zones urbaines qui les jugent plus fiables, plus rapides, plus justes et moins onéreux que les systèmes étatiques.
Le rayonnement de ces systèmes rappelle qu'il est impossible de gérer la pluralité en mettant à l'écart le ou les systèmes indésirables. Le peut-on en imposant un système? L'expérience camerounaise invite à une réponse négative.

\section{B. L'impossibilité d'imposer un système juridique}

Au lendemain de la réunification du Cameroun en 1972, le législateur, pour faire rimer l'unité politique avec l'unité juridique, a entrepris de supprimer la pluralité des systèmes juridiques par l'imposition du système d'origine française. Bien que les intentions du législateur ne soient pas expressément affichées, on les déduit aisément des lieux d'inscription des règles de droit consacrés par la Constitution et de l'analyse du contenu des normes de droit privé adoptées depuis la réunification.

Les lieux d'inscription sont les endroits où chercher les réponses lorsque se pose un problème de droit ${ }^{24}$. Où trouve-t-on les réponses aux problèmes de droit au Cameroun? La Constitution adoptée le 2 juin 1972 au lendemain de la fin de l'État fédéral, après avoir indiqué à l'article 26 les matières qui relèvent du domaine de la loi, dispose à l'article 27 que: «les matières autres que celles qui sont du domaine de la loi ressortissent au pouvoir réglementaire.» L’article 68 ajoute: «la législation des lois et règlements applicables dans l'État fédéral du Cameroun et dans les États fédérés à la date de prise d'effet de la présente Constitution reste en vigueur dans ses dispositions qui ne sont pas contraires aux stipulations de celle-ci, tant qu'elle n'aura pas été modifiée par voie législative ou réglementaire. » Il ressort de ces articles qui n'ont pas été retouchés à l'occasion de la grande révision de la Constitution le 18 janvier $1996^{25}$, que les normes juridiques au Cameroun se trouvent dans les lois ou les règlements.

Cette réponse ne serait aucunement surprenante si on ne se trouvait dans un pays à triple tradition juridique. Sans doute l'absence de référence aux lieux d'inscription des droits traditionnels est-elle une suite logique de la volonté de les supprimer qui anime le législateur depuis l'époque de la colonisation, mais on est tout de même surpris du peu de cas fait à la tradition de la Common Law qui fait de la jurisprudence le principal lieu d'inscription des règles de droit ${ }^{26}$. On se serait en effet

18. C.S. arrêt du 23 avril 1965, Penant, $\mathrm{n}^{\circ}$ 705, 1965, p. 486 ; du 5 mars 1963, Bulletin des arrêts de la Cour suprême du Cameroun, 1965 , p. 543.

19. Cf. S. Melone, «La technique de la codification en Afrique : pratique camerounaise », Revue juridique et politique indépendance et coopération, $\mathrm{n}^{\circ} 3$ et 4 , 1986, p. 307 sq.

20. M. Kamto, «Une justice entre tradition et modernité », p. 57.

21. E. Leroy et M. Wane, «La formation des droits "non étatiques" », p. 358.

22. P. F. Gonidec, Les droits africains. Évolution et sources, Paris, LGDJ, 1968, p. 195.

23. Qui peut être le fait de se partager une noix de cola, de boire l'un après l'autre dans une même coupe ou simplement de se serrer la main. Cf. M. Kamto, «Une justice entre tradition et modernité », p. 58.

24. J. Ghestin, G. Goubeaux, avec le concours de M. Fabre-Magnan, Traité de droit civil. Introduction, $4^{\mathrm{e}}$ édition, Paris, LGDJ, 1994, p. 192 sq.

25. Cf. Loi n 96-o6 du 18 janvier 1996 portant révision de la Constitution du 2 juin 1972 (Yaoundé, Édition SOPECAM, 1996).

26. Cf. E. N. Ngwafor, Family law in Anglophone Cameroon, Regina - Saskatchewan Canada, The University of Regina Press, 1993, p. 1 sq.; R. Cross, Precedent in English law, $3^{\mathrm{e}}$ édition, Oxford, Clarendon Press, 1978 ; R. David et C. Jauffret-Spinosi, Les grands systèmes de droit contemporains, $11^{\mathrm{e}}$ édition, Paris, 
attendu à ce qu'une formule fasse référence, ne serait-ce qu'à titre transitoire, à la jurisprudence comme source de droit. Mais même le titre 5 réservé au pouvoir judiciaire n'en contient pas. En faisant de la loi et du règlement les principaux lieux d'inscription des règles du droit, le constituant camerounais marque sa volonté de substituer le système originaire de la France aux autres. Volonté qu'on peut également déduire du contenu des normes post-réunification.

Depuis la réunification, le Cameroun a adopté plusieurs textes destinés à mettre fin au dualisme juridique antérieur ${ }^{27}$. Certes, dans certains de ces textes, on trouve des préoccupations anglo-saxonnes, mais on se rend vite compte que le législateur les a intégrées pour mieux imposer le système d'origine française. Ainsi, dans le Code du travail qui compte 177 articles, un seul est issu de la Common Law : l'article 24 qui autorise un employeur à prélever directement sur le salaire acquis par un travailleur relevant de son autorité, le montant des cotisations syndicales ordinaires dues par ce dernier, à charge d'en opérer le reversement immédiat à l'organisation syndicale désignée par l'intéressé. De même dans l'ordonnance $\mathrm{n}^{\circ} 72 / 04$ du 26 août 1972, texte de base de l'organisation judiciaire comportant 38 articles $^{28}$, seul l'article 16, devenu article 18 à la faveur de la réforme du 29 décembre 2006 qui reconnaît la compétence du tribunal de grande instance pour les requêtes en "Habeas Corpus », "prohibition» et en « mandamus » ${ }^{29}$ est d'inspiration Common Law.

La volonté d'imposer le système d'inspiration française est plus visible dans le choix des juridictions retenues pour trancher les litiges au premier degré de juridiction. Dans l'ordonnance $\mathrm{n}^{\circ} 72 / 04$ du 26 août 1972 et ses textes modificatifs, sans se soucier de l'organisation judiciaire de type anglo-saxon de l'ex-Cameroun occidental qui opère à ce niveau une distinction entre les Magistrates Courts qui connaissent des petits litiges et des High Courts qui sont des juridictions de droit commun, le législateur a posé que la justice est rendue au nom du peuple camerounais par les tribunaux de grande instance, les tribunaux de première instance, les juridictions de droit traditionnel. La distinction entre les tribunaux de première et grande instance étant faite à partir de la gravité de l'infraction en matière pénale, et de l'objet ou du taux du litige en matière non répressive $e^{30}$. En dehors des juridictions de droit traditionnel dont la compétence peut être déclinée au profit des tribunaux de première et grande instance, il y a reprise du système originaire de la France. Comment le comprendre dans un pays ayant une tradition de Common Law autrement que par la volonté législative d'imposer le système de droit civil?

Cette entreprise d'imposition du droit d'origine française a-t-elle produit les résultats escomptés? Y a-t-il désormais un droit moderne unique d'inspiration française au Cameroun? L'observation révèle une réponse négative.

Sur le fond, les principaux lieux d'inscription des règles de droit dans la partie anglophone du Cameroun restent, malgré les prescriptions de la constitution, prioritairement la jurisprudence ${ }^{31}$. De même, pour les matières ayant fait l'objet d'une législation unique, les juges dans cette partie du pays usent du pouvoir d'interprétation pour s'affranchir des règles du droit civil et appliquer les solutions de la Common Law, des «doctrines of equity» et des "statutes of general application which were in force in England on the first day of january $1900 »^{32}$.

Sur la forme, l'imposition d'une organisation judiciaire unique par les ordonnances de 1972, plusieurs fois modifiées, aurait dû entraîner automatiquement une modification des procédures sur l'étendue du territoire, au minimum de la procédure pénale. Elle devrait entraîner une généralisation de la procédure de type inquisitoire avec un ministère public, partie privilégiée au procès, siégeant à l'estrade à côté du juge, « dominant la partie poursuivie placée en contrebas ${ }^{33}$. Mais la prescription législative n'a eu aucun écho dans la partie anglophone où a été maintenu un système de type accusatoire,

Dalloz, 2002; J. A. Jolowicz, «La jurisprudence en droit anglais : aperçu sur la règle du précédent», in La jurisprudence. Archives de Philosophie du droit, t. 30, 1985, p. 105 sq.

27. On peut citer notamment : sur le fond, un nouveau Code du travail (issu de la loi n ${ }^{\circ} 74 / 14$ du 27 novembre 1974, aujourd'hui remplacé par un autre issu de la loi n 92/007 du 14 août 1992), trois ordonnances : une du 6 juillet 1974 fixant le régime foncier, une autre du 29 juin 1981 portant organisation de l'État civil et diverses dispositions relatives au mariage, et une du 13 décembre 1989 portant sur l'indemnisation des victimes d'accidents de la circulation. Sur la forme, les principaux textes portent sur l'organisation judiciaire. Celui de base est l'ordonnance n $72 / 04$ du 26 août 1972 sur l'organisation judiciaire, plusieurs fois modifiée (voir note 27 ).

28. Ordonnance $\mathrm{n}^{\circ} 72 / 04$ du 26 août 1972 portant organisation judiciaire, modifiée par les ordonnances $\mathrm{n}^{\circ} 72 / 21$ du 19 octobre $1972,73 / 09$ du 25 avril 1973, les lois $n^{\circ} 74 / 03$ du 25 juillet 1974, 76/17 du 8 juillet 1976, 89/019 du 29 décembre 1989, 90/58 du 19 décembre 1990, 98/o08 du 14 avril 1998, 2006/15 du 29 décembre 2006.

29. D'après l'article 16 de l'ordonnance $n^{\circ} 72 / 04$ du 26 août 1972, plusieurs fois modifiée, le tribunal de grande instance est compétent : a) pour connaître des requêtes en libération immédiate formées soit par une personne emprisonnée ou détenue, soit en son nom lorsque lesdites requêtes sont fondées sur un cas d'illégalité formelle ou sur le défaut de titre de détention (Habeas Corpus) [...] ; g) pour connaître de toute requête tendant à obtenir, en matière non administrative, l'interdiction à toute personne ou autorité d'accomplir un acte pour lequel elle est légalement incompétente (prohibition); h) pour connaître des requêtes tendant à obtenir, en matière non administrative, l'accomplissement par toute personne ou autorité, d'un acte qu'elle est tenue d'accomplir en vertu de la loi (mandamus).

30. Les tribunaux de grande instance jugent les crimes et délits connexes, ceux de première instance les délits et contraventions. En matière non répressive, les tribunaux de grande instance jugent en plus les litiges concernant l'État des personnes : État civil, mariage, divorce, filiation, légitimation, adoption, etc. Cf. art. 13 sq. de la loi n 2006-15 du 29 décembre 2006.

31. Cf. E. Ngwafor, Family law in Anglophone Cameroon, p. 28.

32. Section 11 of the Cameroon High Court Law 1955, applicable au Nigeria et dans l'ex-Cameroun occidental.

33. Cf. J. M. Tchakoua, «Des acteurs et procédés dans la nouvelle procédure pénale», Les tendances de la nouvelle procédure pénale camerounaise, J. M. Tchakoua (dir.), Yaoundé, PUA, 2007, p. 7 sq.; V. Tchokomakoua, «Chronique d'une longue gestation: du Code d'instruction criminelle au Code de procédure pénale », in Les tendances de la nouvelle procédure pénale camerounaise, p. 21 sq. 
garantissant l'égalité entre l'accusateur et l'accusé au cours du procès, placés au même niveau dans la salle, organisant un duel judiciaire entre les parties en audience publique avec des débats oraux, publics et contradictoires ${ }^{34}$. En matière de procédure pénale, le système anglo-saxon a fait plus que résister puisqu'il a été généralisé à l'ensemble du territoire par une loi du 25 juillet 2005 instituant un Code de procédure pénale unique pour le Cameroun ${ }^{35}$

Comme on le constate, il est impossible de faire disparaitre la pluralité soit par mise à l'écart d'un ou plusieurs systèmes, soit par imposition de l'un d'eux. Si tel est le cas, c'est que la pluralité, lorsqu' elle existe ou s'introduit dans un pays, devient un élément de son patrimoine juridique; et le meilleur usage à faire d'un patrimoine c'est de l'exploiter : c'est, nous semble-t-il, la voie à explorer.

\section{La voie à explorer : l'exploitation de la pluralité}

Exploiter une chose c'est la faire valoir, en tirer profit. Comment exploiter la pluralité pour gérer les systèmes juridiques? Posée de cette manière, la question n'a pas interpellé les auteurs camerounais qui, pour la plupart, à l'occasion de l'analyse du pluralisme juridique, ont mis l'accent sur un autre chantier important: le point de savoir s'il faut ou non supprimer les droits traditionnels pour construire un droit du développement ${ }^{36}$. Elle nous semble pourtant primordiale si l'on veut élaborer un système juridique sans parti pris idéologique, selon les canons de la science juridique, reflétant les conditions économiques, sociales et culturelles du pays et surtout ayant des chances d'être reçu par ses destinataires. La tâche, sans être impossible, n'est pas aisée. Elle impose de choisir une technique d'exploitation (A) et de la mettre en ouvre (B).

\section{A. Le choix d'une technique d'exploitation de la pluralité}

Deux techniques juridiques sont utilisables pour exploiter la pluralité des systèmes juridiques dans un espace géographique: la juxtaposition et l'harmonisation.
Laquelle doit-on choisir? En mettant en exergue les limites de la juxtaposition, les enseignements de l'expérience camerounaise révèlent le caractère incontournable de l'harmonisation.

Juxtaposer des systèmes juridiques, c'est les mettre côte à côte, c'est attribuer à chaque système son domaine ou sa zone d'influence. On trouve dans un article consacré à la défense du mariage coutumier des propos révélant un soutien incident à cette technique. Pour l'auteur, parce que le droit traditionnel jure en plusieurs de ses aspects avec le droit occidental, il «serait sage de prévoir deux systèmes cohérents et autonomes: un système de règles formant le droit traditionnel dont l'application serait sanctionnée par des juridictions traditionnelles et un système de règles formant le droit moderne d'inspiration occidentale relevant des juridictions modernes ${ }^{37}$. Le Cameroun a utilisé la juxtaposition au lendemain de l'indépendance pour tenir compte de la nature fédérale de l'État. Tandis que dans la partie est était appliqué le système hérité de la France, dans la partie ouest était en vigueur celui hérité de la Grande-Bretagne. L'avènement de l'État unitaire l'ayant rendue inutile, on pensait la technique désormais bannie. Mais en posant la règle: «option de juridiction emporte option de législation» en $1981^{38}$, la Cour suprême a donné l'impression de la ressusciter par la délimitation des zones d'influence des systèmes de droit moderne et des systèmes des droits traditionnels. Mais la haute juridiction a ébranlé sa propre jurisprudence en admettant par deux arrêts rendus successivement en 1985 et en 1987, non contredits jusqu'à aujourd'hui, que les juridictions de droit moderne peuvent valablement utiliser des règles d'origine coutumière ${ }^{39}$. Cette jurisprudence marque un abandon définitif par ce pays de la juxtaposition comme technique de gestion de la pluralité des systèmes juridiques.

De fait, est-il possible dans un État de juxtaposer des règles juridiques sans créer des inégalités et des discriminations? Sans doute est-ce une technique utile dans un État fédéral, mais elle est dangereuse pour un État unitaire car sur le plan politique, elle constitue une menace pour l'unité et la cohésion du pays et sur le plan juridique, elle heurte le caractère général, impersonnel et obligatoire de la règle de droit. La prise en compte de ces réserves rend incontournable l'harmonisation.

34. Dans cette procédure prévue par le «Criminal procedure ordinance » extrait des «Laws of Nigeria », les parties prennent une part importante dans le déroulement de l'audience : elles font citer qui elles veulent comme témoins et les interrogent. L'interrogatoire d'un témoin par la partie qui l'a fait citer est appelé examination in chief. Celui d'un témoin par une partie autre que celle qui l'a cité est appelé cross examination. La reexamination entendue comme l'interrogatoire d'un témoin après cross examination étant également permise.

35. Loi n ${ }^{\circ} 2005 / 007$ du 27 juillet 2005 portant Code de procédure pénale, édition AES, Yaoundé, PUA, 2005.

36. Cf. notamment B. Banamba, Les conflits de droits et de lois dans le système juridique camerounais (droit de la famille et des personnes), Thèse de doctorat en droit, Paris II, 1993; P. G. Pougoue, La famille et la terre: essai de contribution à la systématisation du droit privé au Cameroun, Thèse de doctorat d'État en droit, Bordeaux, 1977; A. D. Tjouen, Droits domaniaux et techniques foncières en droit camerounais (étude d'une réforme législative), Paris, Economica, 1982 ; S. Melone, "Du bon usage du pluralisme judiciaire en Afrique »; "La technique de la codification en Afrique : pratique camerounaise ", RJPIC, $\mathrm{n}^{\circ} 3$ et $4,1986, \mathrm{p} .306$ sq.

37. S. Ombiono, «Le mariage coutumier en droit camerounais», p. 32.

38. Proclamée en 1981 pour casser un arrêt de cour d'appel admettant un divorce prononcé par un juge de droit moderne accueillant une cause coutumière de divorce, la règle signifie que les juridictions de droit moderne ne doivent appliquer que le droit d'inspiration étrangère et celles de droit traditionnel, le droit coutumier. C.S. arrêt n ${ }^{\circ}$ 28/CC du 10 décembre 1981 (affaire Ango Parfait c. Beyidi Pauline) dans F. Anoukaha (dir.), Les grandes décisions de la jurisprudence civile camerounaise, Yaoundé, LERDA, 2008, p.85 sq., obs. F. Anoukaha.

39. Affaire Kemajou, C.S. Arrêt $n^{\circ} 86 / \mathrm{CC}$ du 18 juillet 1985, Revue juridique africaine, $\mathrm{n}^{\circ} 3,1990$, p. 75 sq.; affaire Koum, C.S. arrêt $\mathrm{n}^{\circ}$ 64/CC du 16 juillet 1987, ibid., note F. Anoukaha; Juridis info, n 3 (spécial), 1990, p. 99 sq., obs. L. Elomo Ntonga. 
L'harmonisation est une technique juridique qui permet d'unifier les ensembles législatifs ou des systèmes juridiques différents par élaboration d'un droit nouveau empruntant aux uns et aux autres. Cette technique a fait la preuve de son efficacité ailleurs ${ }^{40}$ et récemment encore dans l'ordre international ${ }^{41}$, elle peut également être utile dans l'ordre interne.

C'est une technique qui n'a jamais eu les faveurs de la doctrine camerounaise ${ }^{42}$. On a notamment soutenu qu'elle ne peut permettre de former un droit unique et homogène à partir des droits traditionnels et des droits occidentaux différents en tous points ${ }^{43}$. L'argument qui vise surtout à sauver les droits traditionnels est surprenant car c'est la différence des systèmes qui permet d'envisager l'harmonisation. L'harmonisation vise justement à faire un système nouveau à partir des systèmes différents. C'est en réalité la seule qui peut aider à l'élaboration d'un droit prenant en compte les systèmes de droits traditionnels, et les systèmes de droit moderne dans une symbiose permettant l'éclosion d'un droit reflétant les véritables aspirations et contraintes du Cameroun ${ }^{44}$. Cependant, elle est difficile à mettre en œuvre.

\section{B. Mise en œuvre de la technique choisie}

Comment harmoniser de manière efficace et utile? Comment articuler des systèmes aussi disparates pour en faire émerger un, cohérent, reflétant les aspirations et contraintes d'un pays? La prise en compte de quatre exigences majeures nous semble susceptible de permettre d'atteindre ce résultat: le respect des engagements internationaux du pays, le souci d'effectivité, d'accessibilité et d'intelligibilité du droit.

Le respect des engagements internationaux est le socle sur lequel doit à notre sens être harmonisé le droit d'un pays. Dans le préambule de la Constitution du Cameroun $^{45}$, on peut lire : «le peuple camerounais [...] affirme son attachement aux libertés fondamentales inscrites dans la Déclaration universelle des Droits de l'homme, la Charte des Nations unies, la Charte africaine des Droits de l'homme et des peuples et toutes les conventions internationales y relatives. » Ces textes contiennent des valeurs et principes juridiques dont le respect peut aider à harmoniser de manière efficace. Il en est ainsi du souci de préservation de la dignité de la personne humaine, des principes d'égalité et de non-discrimination notamment. Leur prise en compte peut notamment aider à dépouiller les systèmes de droit traditionnel de toutes les règles et procédures portant atteinte aux droits et libertés de la personne humaine, pour laisser subsister celles qui peuvent entrer dans le système harmonisé. Il devrait notamment conduire à uniformiser le système judiciaire dont la distinction entre les juridictions de droit moderne et les juridictions de droit traditionnel est considérée comme le symbole de la discrimination et des inégalités ${ }^{46}$. Il peut également permettre de faire le tri pour récupérer des règles et procédures dans les systèmes étrangers.

Le souci d'effectivité du droit est l'autre exigence qui doit être prise en compte dans l'harmonisation. Au cœur de la relation entre droit et société ${ }^{47}$, le concept d'effectivité, originaire de la sociologie du droit, appartient désormais au langage des juristes ${ }^{48}$. D'après le Vocabulaire juridique de l'Association Henri Capitant, il désigne «le caractère d'une règle de droit qui produit l'effet voulu, qui est réellement appliquée ${ }^{49}$. Cette définition a été critiquée par la doctrine qui trouve qu'elle assimile effectivité et efficacité ${ }^{50}$. De fait, c'est le degré de réalisation dans les pratiques sociales du droit qui caractérise l'effectivité. Un système de droit effectif est donc celui qui est réellement appliqué. Articuler les systèmes de droit traditionnel et les systèmes de droit moderne en vigueur au Cameroun pour produire un système unique ayant plus de chances d'être réellement appliqué: telle est une des exigences qui doivent guider l'harmonisation des systèmes juridiques au Cameroun. Sa prise en compte permet d'éviter d'imposer des solutions qui heurtent de front les convictions et les pratiques des populations et partant de mettre en place un système ayant des chances d'être réellement appliqué. Dès lors que le respect des engagements internationaux aura permis de dépouiller les systèmes de droit traditionnel de leurs aspects contraires à la dignité de la personne humaine et aux droits fondamentaux en général, on devrait y puiser abondamment les éléments de construction du nouveau système, notamment ceux qui sont communs à un maximum de tribus ou à des

40. Le Code Civil de 1804 en France en est l'illustration majeure. Cf. R. Cabrillac, Les codifications, Paris, PUF (Droit et société), 2002, p. 154 sq.

41. Elle a été récemment utilisée pour créer un système juridique commun aux États membres de l'organisation pour l'harmonisation du droit des affaires en Afrique (OHADA).

42. Notamment S. Melone, «Du bon usage du pluralisme judiciaire en Afrique »; S. Ombiono, «Le mariage coutumier en droit camerounais ».

43. Ibid., p. 32 .

44. Sur la nécessité de créer un droit camerounais, lire notre article «Doctrine et création du droit privé au Cameroun ", Revue de la recherche juridique. Droit prospectif, $\mathrm{n}^{\circ} 4,2005$, p. 2455 sq.

45. Constitution du Cameroun, Loi n 96-06 du 18 janvier 1996. L'article 65 de ce préambule fait partie intégrante de la Constitution.

46. B. Bananba, « Regard nouveau sur un texte déjà trentenaire : le cas du Décret du 19 décembre 1969 portant organisation et fonctionnement des juridictions traditionnelles de l'ex-Cameroun oriental», p. 128-129.

47. Cf. J. Carbonnier, «Effectivité et ineffectivité de la règle de droit », L’Année sociologique, 1958, p. 3 sq. Texte repris dans Flexible droit, Paris, LGDJ, 1992; J. Commaille, «Effectivité», in Dictionnaire de la culture juridique.

48. Cf. A. Jeammaud, «Le concept d'effectivité en droit », in L'effectivité du droit du travail: à quelles conditions?, P. Auvergnon (dir.), Actes du séminaire international de droit comparé des relations professionnelles et de la Sécurité sociale, Bordeaux, COMPTRASEC UMR CNRS - Université Bordeaux IV, 2006, p. 33 sq.

49. Vocabulaire juridique de l'Association Henri Capitant, p. 345-346.

50. Notamment A. Jeammaud, «Le concept d'effectivité en droit », p. 47 sq. 
régions. Ils auront d'autant plus de chances d'être appliqués que les utilisateurs s'y reconnaîtront. En cas de silence, d'insuffisance des solutions traditionnelles, on devra recourir à celles des droits étrangers qui sont appliquées sans grandes difficultés depuis leur instauration. C'est la tâche de la doctrine de faire émerger ces éléments de construction. Elle est sans doute ardue, mais elle est inévitable et urgente, car c'est la voie de construction d'un système juridique ayant des chances de refléter les caractéristiques et les aspirations du pays ${ }^{51}$.

L'harmonisation doit enfin tenir compte des soucis d'accessibilité et d'intelligibilité du droit nouveau, autrement dit du choix d'une forme de présentation du droit. La forme orale privilégiée par les droits traditionnels africains, tout comme la construction progressive du droit par la jurisprudence étant disqualifiées, la réunion en codes des règles de droit désignée techniquement codification devient incontournable.

L'idée d'utiliser la codification comme technique de mise en forme du droit n'est pas nouvelle en Afrique noire en général ${ }^{52}$ et au Cameroun en particulier. Encore faut-il déterminer le type de codification nécessaire. D'après le Vocabulaire juridique de l'Association Henri Capitant, ce mot qui désigne à la fois l'action de faire un code et le résultat de cette action renvoie à deux opérations distinctes. Une qui consiste à rassembler, fixer, clarifier, rénover, systématiser ou unifier les règles relatives à une ou plusieurs matières en les ordonnant en un ou plusieurs codes qu'on appelle codification « réelle» ou à droit nouveau, et une autre qui consiste à mettre en un document des textes préexistants reclassés selon leur nature, non modifiés en substance, ou à réunir en un code, moyennant les modifications de forme nécessaires, mais à l'exclusion de toute modification de fond, de toutes les dispositions existantes en la matière qu'on appelle codification à droit constant ${ }^{53}$. Seule la codification « réelle» ou à droit nouveau permet d'harmoniser. C'est elle qui permet de fondre en un droit unique les règles traditionnelles, celles d'inspiration française et les normes relevant de la Common Law choisies comme droit camerounais nouveau.

De l'expérience camerounaise, il ressort que la pluralité des systèmes juridiques introduite dans les États d'Afrique noire par les colons est devenue un élément de leur patrimoine que l'on ne peut plus supprimer, mais seulement chercher à exploiter. Il en ressort également que la juxtaposition, technique d'exploitation la plus simple, est également la plus incommode et qu'il faut l'éviter pour privilégier l'harmonisation qui doit être mise en œuvre en tenant compte des engagements internationaux du pays, du souci d'effectivité, d'accessibilité et d'intelligibilité du droit.

Si cette recherche peut aider les États à plusieurs traditions juridiques à mieux gérer la pluralité de leurs systèmes juridiques, l'objectif que nous poursuivions en l'entreprenant serait atteint.

51. On pourrait, par exemple, dans un système judiciaire désormais unifié, instituer une obligation de conciliation préalable obligatoire à tout procès pour tenir compte à la fois des exigences africaines de réconciliation et de celles occidentales de détermination des droits individuels des justiciables. Sur cette opposition d'enjeu, voir M. Kamto, «Une justice entre tradition et modernité», p. 58.

52. Cf. R. Degni-Segui, «Codification et uniformisation du droit », in Encyclopédie juridique de l'Afrique, t. 1, L'État et le droit, p. 455 sq.

53. Cf. Vocabulaire juridique de l'Association Henri Capitant, p. 167. Lire également R. Cabrillac, Les Codifications, Paris, PUF (Droit, éthique et société), 2002, p. 55 sq. 